\title{
Complete Genome Resources for Ralstonia Bacterial Wilt Strains UW763 (Phylotype I); Rs5 and UW700 (Phylotype II); and UW386, RUN2474, and RUN2279 (Phylotype III)
}

\author{
Olivia R. Steidl, ${ }^{1}$ Alicia N. Truchon, ${ }^{1,2}$ Madeline M. Hayes, ${ }^{1,2}$ and Caitilyn Allen ${ }^{1, \dagger}$ \\ ${ }^{1}$ Department of Plant Pathology, University of Wisconsin-Madison, Madison, WI 53706, U.S.A. \\ ${ }^{2}$ Microbiology Doctoral Training Program, University of Wisconsin-Madison, Madison, WI 53706, \\ U.S.A.
}

\begin{abstract}
We share whole genome sequences of six strains from the Ralstonia solanacearum species complex, a diverse group of Betaproteobacteria that cause plant vascular wilt diseases. Using single-molecule real-time technology, we sequenced and assembled full genomes of Rs5 and UW700, two phylotype IA-sequevar 7 (IIA-7) strains from the southeastern United States that are closely related to the $R$. solanacearum species type strain, $\mathrm{K} 60$, but were isolated $>50$ years later. Four sequenced strains from Africa include a soil isolate from Nigeria (UW386, III-23), a tomato isolate from Senegal (UW763, I-14), and two potato isolates from the Madagascar highlands (RUN2474, III-19 and RUN2279, III-60). This resource will support studies of the genetic diversity, ecology, virulence, and microevolution of this globally distributed group of high-impact plant pathogens.
\end{abstract}

Plant pathogens in the Ralstonia solanacearum species complex (RSSC) cause bacterial wilt disease, a major threat to global food security (Mansfield et al. 2012). R. solanacearum strains infect more than 250 different host plants from around the world, including crops vital to both subsistence farming and commercial agriculture such as potato, banana, and tomato (Allen et al. 2005).

The broad $R$. solanacearum host range is likely due to the enormous heterogeneity of its 5.1- to 5.8-MB genome (Genin and Denny 2012). $R$. solanacearum is so phenotypically and genetically diverse that it is classified as a species complex, with only approximately $50 \%$ of genes conserved across the complex (Ailloud et al. 2015; Remenant et al. 2010). The RSSC is subdivided into four phylotypes that correlate with geographic origin; strains are further separated into sequevars based on the endoglucanase (egl) gene sequence (Fegan and Prior 2005; Prior and Fegan 2005). R. solanacearum strains sequenced to date carry 4,600 to 5,200 coding sequences with a shared (core) genome of approximately 2,400 unigenes ( $P$. Sharma and B. Vinatzer, unpublished). Average nucleotide identity (ANI) analysis shows that these conserved core genes are, on average, $>96 \%$ identical among strains in the same phylotype, while core genes from strains in different phylotypes are 91 to 95\% identical (Remenant et al. 2011). The RSSC pangenome contains at least 10,000 genes (P. Sharma and B. Vinatzer, unpublished). This large set of variable genes likely encodes some of the group's diversity in host range, optimum virulence temperature, and other biological traits relevant to its life as a pathogen.

${ }^{\dagger}$ Corresponding author: C. Allen; callen@wisc.edu

The author(s) declare no conflict of interest.

Accepted for publication 4 July 2021.

\section{Funding}

United States Department of Agriculture Hatch grant MSN222769 (to C. Allen), University of WisconsinMadison Hilldale Research Fellowship (to O. R. Steidl), and the University of Wisconsin-Madison College of Agricultural and Life Sciences.

\section{Keywords}

bacterial wilt, comparative genomics, Madagascar, Nigeria, potato brown rot, Ralstonia solanacearum, Ralstonia solanacearum species complex, Senegal 
Table 1. Characteristics of genome sequences from seven strains in the Ralstonia solanacearum species complex, including $\mathrm{K}^{\circ} \mathrm{O}^{\top}$, the R. solanacearum type strain ${ }^{a}$

\begin{tabular}{|c|c|c|c|c|c|c|c|c|c|}
\hline Strain ID & Phyl-Seq ${ }^{b}$ & Host, location & $\begin{array}{l}\text { Total } \\
\text { length }\end{array}$ & $\begin{array}{l}\text { Chr } \\
\text { length }\end{array}$ & $\begin{array}{l}\text { Plasmid } \\
\text { length }\end{array}$ & $\mathrm{CDS}^{\mathrm{c}}$ & $\begin{array}{l}\text { rRNA } \\
\text { genes }\end{array}$ & $\begin{array}{l}\text { tRNA } \\
\text { genes }\end{array}$ & Bioproject ID ${ }^{d}$ \\
\hline UW763 & $\mathrm{I}-14$ & Tomato, Senegal & $5,783,389$ & $3,762,104$ & $2,021,285$ & 5,004 & 12 & 58 & PRJNA591018 9 \\
\hline $\mathrm{Rs5}^{\mathrm{e}}$ & IIA-7 & $\begin{array}{c}\text { Tomato, Florida, } \\
\text { United States }\end{array}$ & $5,430,180$ & $3,592,797$ & $1,837,383$ & 4,739 & 9 & 56 & PRJNA591018 9 \\
\hline UW700 & IIA-7 & $\begin{array}{c}\text { Tomato, Virginia, } \\
\text { United States }\end{array}$ & $5,487,557$ & - & - & 4,952 & 9 & 61 & PRJNA381968 \\
\hline $\mathrm{K} 60^{\top}$ & IIA-7 & $\begin{array}{l}\text { Tomato, North } \\
\text { Carolina, United } \\
\text { States }\end{array}$ & $5,770,663$ & $3,383,865$ & $1,931,798$ & 4,551 & 9 & 55 & PRJNA381968 \\
\hline UW386 & III-23 & Soil, Nigeria & $5,488,379$ & $3,658,282$ & $1,830,097$ & 4,787 & 12 & 57 & PRJNA531204 \\
\hline RUN2279 ${ }^{f}$ & III-60 & Potato, Madagascar & $5,746,272$ & $3,835,000$ & $1,911,272$ & 5,152 & 12 & 56 & PRJNA591018 9 \\
\hline RUN2474 & III-19 & Potato, Madagascar & $5,654,469$ & $3,712,138$ & $1,942,331$ & 5,109 & 12 & 59 & PRJNA591018 9 \\
\hline
\end{tabular}

${ }^{\mathrm{a}} \mathrm{Chr}=$ chromosome, $\mathrm{rRNA}=$ ribosomal RNA, and tRNA = transfer RNA.

${ }^{\mathrm{b}}$ For each strain, phylotype was determined using the phylotype multiplex PCR (Fegan and Prior 2005) and sequevar was determined based on nucleotide sequence similarity of a 750-bp internal fragment of the endoglucanase (egl) gene (Poussier et al. 2000).

${ }^{\mathrm{c}} \mathrm{CDS}=$ predicted coding sequences.

${ }^{\mathrm{d}}$ Bioproject IDs refer to genome sequences deposited in the open access NCBI database.

e Alternate strain name: UW576.

${ }^{f}$ Alternate strain name: UW773.

${ }^{g}$ Alternate strain name: UW774.

Genome sequences of varying quality are available for several hundred $R$. solanacearum strains (Lowe-Power and Chipman 2020). However, South American (phylotype IIB) and Asian (phylotype I) strains are overrepresented, whereas there are relatively few sequences of strains from North America (phyl IIA), Africa (phylotype III), and Indonesia and Japan (phylotype IV). Therefore, we selected six $R$. solanacearum strains from these underrepresented groups: two isolated in the southeastern United States and four isolated on the African continent (Table 1).

Bacterial DNA was extracted using the Epicentre MasterPure Kit (Middleton, WI, U.S.A.). PacBio Sequel II long-read sequencing was conducted at Great Lakes Genomics Center at University of Wisconsin-Milwaukee for UW386 and UW700, and at NovoGene (Sacramento, CA, U.S.A.) for Rs5, UW763, RUN2279, and RUN2474. This generated 54,382 reads for UW386, 61,674 reads for UW700, 19,278 reads for UW576, 23,957 for UW763, 27,388 for UW773, and 20,132 reads for UW774. This input generated coverage of 340x for UW386 and UW700, 140x for UW576, 165x for UW763, 190x for UW773, and 142x for UW774. Reads for UW386 were assembled using HGAP5 (Chin et al. 2013). Reads for UW700, UW576, UW763, UW773, and UW774 were assembled using Canu version 1.9 (randomly downsampled to $32 \times, 39 \times, 35 \times, 37 \times$, and $37 \times$, respectively), circularized using Circlator version 1.5.5, and annotated through the NCBI Prokaryotic Genome Annotation Pipeline (Hunt et al. 2015; Koren et al. 2017; Tatusova et al. 2016). $\mathrm{N}_{50}$ S for these assemblies were 3,658,282, 1,697,113, 3,592,797, 3762104, 3,835,000, and 3,712,138 bp with benchmarking universal single-copy ortholog (BUSCO) complete values of 100.0, 99.5, 99.8, 99.8, 100.0, and $100.0 \%$ for UW386, UW700, UW576, UW763, UW773, and UW774, respectively. These assembly completeness metrics were calculated using gVolante2, sourcing BUSCO v5 and its corresponding Betaproteobacteria ODBv10 database of 569 genes (Nishimura et al. 2019; Seppey et al. 2019).

Genomes of all strains except for UW700 were closed and contained two contigs corresponding to the two circular replicons typically present in RSSC strains (Genin and Denny 2012). Although neither replicon is dispensable, the longer element is commonly known as the chromosome and the smaller element is known as the megaplasmid. An updated UW700 genome assembly has replaced the previous 18-conting version assembled using HGAP5. The total contigs were reduced to 11 by assembling the raw PacBio reads with Canu version 1.9. Percent ANI between genomes was determined based on orthologous sequences, as described by Yoon et al. (2017).

The $R$. solanacearum type strain, $\mathrm{K} 60^{\top}$, isolated from tomato in Raleigh, NC in 1953, belongs to North American phylotype IIA, sequevar 7, abbreviated IIA-7 (Hayes et al. 2017; Kelman 1954). We sequenced two more IIA-7 strains: Rs5 and UW700. Rs5, also known as 
UW576, was isolated from tomato in 2000 in Quincy, FL (Ji et al. 2007). UW700 was isolated from tomato on the Eastern Shore of Virginia in 2010 (Wimer et al. 2011). These strains share a host, sequevar, and geographic origin with $\mathrm{K} 60^{\top}$ but were isolated approximately 50 years later; thus, they offer an opportunity to observe microevolution and identify genes recently under selection (Castillo and Agathos 2019; Hedge and Wilson 2016). The genome of Rs5 has $99.68 \%$ ANI with both $\mathrm{K}^{\circ} 0^{\top}$ and UW700, and UW700 has a $99.71 \%$ ANI with K60. MAUVE alignments of sequences from these three strains found no major wholegenome rearrangements, consistent with a recent common ancestor.

A second group of sequenced strains belongs to African phylotype III, including UW386 (III-23); RUN2279, also known as UW773 (III-60); and RUN2474, also known as UW774 (III-19). UW386 was isolated in 1979 in Nigeria from soil and RUN2279 and RUN2474 were isolated from potato in 2013 in the highlands of Madagascar (Ravelomanantsoa et al. 2018). Although the two Madagascar strains are in different sequevars, their shared genes have 99.3\% ANI. In contrast, RUN2279 and RUN2474 share only 96.6\% ANI with Nigerian soil isolate UW386, close to the approximately $96 \%$ ANI cut-off used to separate phylotypes (Remenant et al. 2010).

A fourth strain isolated in Africa, UW763, came from a wilting tomato plant in Senegal in 2017 but belongs to Asian phylotype I-14. The UW763 genome shares $99.3 \%$ ANI with that of the well-studied phylotype I-18 strain GMI1000.

This set of genome resources will support studies of the genetic diversity, ecology, virulence, and microevolution of this globally distributed group of heterogenous, high-impact plant pathogens.

\section{Acknowledgments}

We thank P. Sharma and B. Vinatzer (Virginia Tech University) for sharing unpublished data and A. L. Perez-Quintero (Colorado State University) and J. M. Jacobs (Ohio State University) for advice and helpful discussions.

\section{Literature Cited}

Ailloud, F., Lowe, T., Cellier, G., Roche, D., Allen, C., and Prior, P. 2015. Comparative genomic analysis of Ralstonia solanacearum reveals candidate genes for host specificity. BMC Genomics 16:270.

Allen, C., Prior, P., and Hayward, A. C., eds. 2005. Bacterial Wilt Disease and the Ralstonia solanacearum Species Complex. American Phytopathological Society, St Paul, MN, U.S.A.

Castillo, J. A., and Agathos, S. N. 2019. A genome-wide scan for genes under balancing selection in the plant pathogen Ralstonia solanacearum. BMC Evol. Biol. 19:123.

Chin, C. S., Alexander, D. H., Marks, P., Klammer, A. A., Drake, J., Heiner, C., Clum, A., Copeland, A., Huddleston, J., Eichler, E. E., Turner, S. W., and Korlach, J. 2013. Nonhybrid, finished microbial genome assemblies from long-read SMRT sequencing data. Nat. Methods 10:563-569.

Fegan, M., and Prior, P. 2005. How complex is the "Ralstonia solanacearum species complex"? Pages 449-461 in: Bacterial Wilt Disease and the Ralstonia solanacearum Species Complex. C. Allen, P. Prior, and A. C. Hayward, eds. American Phytopathological Society, St Paul, MN, U.S.A.

Genin, S., and Denny, T. P. 2012. Pathogenomics of the Ralstonia solanacearum species complex. Annu. Rev. Phytopathol. 50:67-89.

Hayes, M. M., Maclntyre, A. M., and Allen, C. 2017. Complete genome sequences of the plant pathogens Ralstonia solanacearum type strain K60 and $R$. solanacearum Race 3 biovar 2 strain UW551. Genome Announce. 5 : e01088-17.

Hedge, J., and Wilson, D. J. 2016. Practical approaches for detecting selection in microbial genomes. PLOS Comput. Biol. 12:e1004739.

Hunt, M., De Silva, N., Otto, T. D., Parkhill, J., Keane, J. A., and Harris S. R. 2015. Circlator: Automated circularization of genome assemblies using long sequencing reads. Genome Biol. 16:294.

Ji, P., Allen, C., Sanchez-Perez, A., Yao, J., Elphinstone, J. G., Jones, J. B., and Momol, M. T. 2007. New diversity of Ralstonia solanacearum strains associated with vegetable and ornamental crops in Florida. Plant Dis. 91: 195-203.
Kelman, A. 1954. The relationship of pathogenicity of Pseudomonas solanacearum to colony appearance in tetrazolium medium. Phytopathology 44:693-695.

Koren, S., Walenz, B. P., Berlin, K., Miller, J. R., Bergman, N. H., and Phillippy, A. M. 2017. Canu: Scalable and accurate long-read assembly via adaptive $k$-mer weighting and repeat separation. Genome Res. 27:722-736.

Lowe-Power, T. M., and Chipman, K. 2020. A meta-analysis of the known global distribution and host range of the Ralstonia species complex. bioRxiv. https://www.biorxiv.org/content/10.1101/2020.07.13.189936v2

Mansfield, J., Genin, S., Magori, S., Citovsky, V., Sriariyanum, M., Ronald, P., Dow, M., Verdier, V., Beer, S. V., Machado, M. A., Toth, I., Salmond, G., and Foster, G. D. 2012. Top 10 plant pathogenic bacteria in molecular plant pathology. Mol. Plant Pathol. 13:614-629.

Nishimura, O., Hara, Y., and Kuraku, S. 2019. Evaluating genome assemblies and gene models using gVolante. Methods Mol. Biol. 1962:247-256.

Poussier, S., Prior, P., Luisetti, J., Hayward, C., and Fegan, M. 2000. Partial sequencing of the $h r p B$ and endoglucanase genes confirms and expands the known diversity within the Ralstonia solanacearum species complex. Syst. Appl. Microbiol. 23:479-486.

Prior, P., and Fegan, M. 2005. Recent developments in the phylogeny and classification of Ralstonia solanacearum. Acta Hortic. 695:127-136.

Ravelomanantsoa, S., Vernière, C., Rieux, A., Costet, L., Chiroleu, F., Arribat, S., Cellier, G., Pruvost, O., Poussier, S., Robène, I., Guérin, F., and Prior, P. 2018. Molecular epidemiology of bacterial wilt in the Madagascar highlands caused by Andean (Phylotype IIB-1) and African (Phylotype III) Brown Rot strains of the Ralstonia solanacearum species complex. Front. Plant Sci. 8:2258.

Remenant, B., de Cambiaire, J.-C., Cellier, G., Jacobs, J. M., Mangenot, S., Barbe, V., Lajus, A., Vallenet, D., Medigue, C., Fegan, M., Allen, C., and Prior, P. 2011. Ralstonia syzygii, the blood disease bacterium and some Asian $R$. solanacearum strains form a single genomic species despite divergent lifestyles. PLoS One 6:e24356.

Remenant, B., Coupat-Goutaland, B., Guidot, A., Cellier, G., Wicker, E., Allen, C., Fegan, M., Pruvost, O., Elbaz, M., Calteau, A., Salvignol, G., Mornico, D., 
Mangenot, S., Barbe, V., Médigue, C., and Prior, P. 2010. Genomes of three tomato pathogens within the Ralstonia solanacearum species complex reveal significant evolutionary divergence. BMC Genomics 11:379.

Seppey, M., Manni, M., and Zdobnov, E. M. 2019. BUSCO: Assessing genome assembly and annotation completeness. Methods Mol. Biol. 1962:227-245.

Tatusova, T., DiCuccio, M., Badretdin, A., Chetvernin, V., Nawrocki, E. P., Zaslavsky, L., Lomsadze, A., Pruitt, K. D., Borodovsky, M., and Ostell, J. 2016.
NCBI prokaryotic genome annotation pipeline. Nucleic Acids Res. 44:66146624

Wimer, A. F., Rideout, S. L., and Freeman, J. H. 2011. Temporal and spatial distribution of tomato bacterial wilt on Virginia's Eastern Shore. HortTechnology 21:198-201.

Yoon, S. H., Ha, S. M., Lim, J., Kwon, S., and Chun, J. 2017. A large-scale evaluation of algorithms to calculate average nucleotide identity. Antonie Leeuwenhoek 110:1281-1286. 